Santa Clara University

Scholar Commons

Biology

College of Arts \& Sciences

4-2007

\title{
On new characters of the eggs of Embioptera with the description of a new species of Saussurembia (Anisembiidae)
}

Janice Edgerly-Rooks

SantaClara University, jedgerlyrooks@scu.edu

Claudia Szumik

Chanel McCreedy

Follow this and additional works at: http://scholarcommons.scu.edu/bio

Part of the Entomology Commons

\section{Recommended Citation}

EDGERLY, J. S., SZUMIK, C. A., \& MCCREEDY, C. N. (2007). On new characters of the eggs of Embioptera with the description of a new species of Saussurembia (Anisembiidae). Systematic Entomology, 32(2), 387-395

This article is the copyright property of the Entomological Society of America and may not be used for any commercial or other private purpose without specific written permission of the Entomological Society of America.

This Article is brought to you for free and open access by the College of Arts \& Sciences at Scholar Commons. It has been accepted for inclusion in Biology by an authorized administrator of Scholar Commons. For more information, please contact rscroggin@scu.edu. 


\title{
On new characters of the eggs of Embioptera with the description of a new species of Saussurembia (Anisembiidae)
}

\author{
J A N ICE S. EDGERLY ${ }^{1}$, CLAUDIA A. SZUMIK ${ }^{2}$ and \\ CHANEL N. MCCREEDY ${ }^{1}$ \\ ${ }^{1}$ Department of Biology, Santa Clara University, Santa Clara, California, U.S.A. and ${ }^{2}$ CONICET-Instituto Superior \\ de Entomología, Tucumán, Argentina
}

\begin{abstract}
Nine egg characters for eleven species of Embioptera are described for the first time. Egg variability indicates its potential use in taxonomy. Here, we analyse the correlation of this variability with the ordinal classification. In addition, a new species of Saussurembia (Anisembiidae) from Trinidad is described, including classical traits in the systematics of Embioptera (adult male and female), as well as general features of the egg and maternal behaviour.
\end{abstract}

\section{Introduction}

The order Embioptera, also called Embiodea or Embiidina, is well defined by numerous morphological, developmental and behavioural traits (see Grimaldi \& Engel, 2005 for a discussion of the order's name). Most striking is the presence of silk glands in the foretarsi and the ability of all individuals (juvenile and adult, male and female) to spin silk. Females are juvenile in form, retaining a streamlined, wingless body throughout their life. Embiids also exhibit primitively social behaviour in which adult females provide the maternal care of eggs and nymphs and many species form large colonies of individuals that share silk dwellings. Males, mostly winged, generally die soon after mating and do not contribute to the care of their offspring.

The streamlined, nymphoid form of females allows them to traverse narrow silk passages, tight bark crevices or subterranean tunnels. The apparent constraints imposed by their lifestyle have left female embiids without obvious species-specific, genus- or even family-level traits. As a consequence, taxonomists traditionally concentrate on describing male secondary sexual traits and wings in species diagnoses. Here, we provide a description of a colourful new species whose unusual features allow female diversity and evolution to be brought into sharper focus. The paper is divided into two parts. A species description is provided in Part 1, in which female morphology, eggs and behavioural

Correspondence: Janice Edgerly, Department of Biology, Santa Clara University, Santa Clara, CA 95053, U.S.A. E-mail: jedgerlyrooks@scu.edu traits are added to the male and female traits usually featured in species descriptions (e.g. Szumik, 1998). In Part 2 , the informative nature of embiid egg variability in embiid classification is explored.

\section{Part 1. A new species from Trinidad, West Indies}

\section{Materials and methods}

All measurements are given in millimetres. The ocular ratio (OR) is defined in Szumik (1991). Features of the wing base union are given in Szumik (1996). The following abbreviations have been used: 10Lp1, caudal process of the tenth left hemitergite; 10Rp1, caudal process of the tenth right hemitergite; $10 \mathrm{~T}$, tenth tergite; Ep, epiproct; $\mathrm{H}$, hypandrium; $\mathrm{Hp}$, process of the hypandrium; LC1, left basal cercus; LC2, left apical cercus; Lpp, left paraproct; Rpp, right paraproct; Mm, mentum; Sm, submentum; Md, mandible. The terminology used to describe the chaetotaxy of the hind basitarsus involves the number of setae followed by a letter denoting the area in which they are present ( $\mathrm{P}$, prolateral; $\mathrm{R}$, retrolateral; $\mathrm{V}$, ventral); two numbers and a letter (A, apical; B, basal) in parentheses indicate the segment ratio. For example, $10 \mathrm{P}(1: 2 \mathrm{~A})$ indicates ten setae in the apical half of the prolateral area.

\section{Results and discussion}

The character combination of the new species indicates that it is closely related to the anisembiids Stenembia Ross 
1972 and Saussurembia Davis 1939. Unfortunately, the limits, as well as the concepts, of these genera remain unclear. Many of the diagnostic characters used for Stenembia also occur in Saussurembia, i.e. Md acute and small, LC1 symmetrical (processes and setae absent), Hp elongate, Ep narrow (stick-like), wing venation ( $\mathrm{Rs}+\mathrm{Ma}$ and $\mathrm{Rs}$ are the only veins sclerotized and cross veins are absent except between R1 and Rs) and the general shape of 10Lpl and 10Rp1 (with small differences in size and degree of sclerotization). Those characters for which these genera differ indicate that one is paraphyletic with respect to the other, and that Saussurembia is the autapomorphic form of this group (Szumik et al., 2003). Moreover, the new species has a combination of characters of Saussurembia and Stenembia. The new species and Saussurembia share the presence of a sclerotized line starting from the inner margin of $10 \mathrm{Lp} 1$, the ventrally curved $10 \mathrm{Rp} 1$ and the basally broad 10Lp1 with acute apex. The new species and Stenembia have in common the well-defined Lpp and Rpp, the dorsally curved Lpp and the directed leftward Hp. Because Saussurembia was described first and a formal synonymization with Stenembia is inevitable, the new species is placed in Saussurembia.

\section{Saussurembia calypso new species}

Etymology. The specific name derives from the Greek calyps meaning 'beautiful nymph' (Borror, 1960). The name refers to the colourful appearance and to the discovery of this species in Trinidad, the home of the popular form of music called 'calypso'.
Diagnosis. Saussurembia calypso differs from the other species of Stenembia and Saussurembia by having Rpp dorsally prolonged, 10Lp1 totally covered by Lpp, Hp elongated and with the acute apex appearing as a second process of 10Rp1 and Md without molar teeth.

Male (holotype). Colour: head dark brown, clypeal region with two small elliptical less pigmented areas. Antenna: first antennite dark brown, the rest lighter brown. Prothorax and basal cerci brownish white; apical cerci lighter brown. Meso, metathorax and legs lighter brown. Abdomen lighter reddish-brown with some reddish pigmentation.

Total length: 7.30. Features of the head, chaetotaxy and terminalia are shown in Fig. 1A-E. Head discoidal: width/ length: 0.95. OR: 0.44. Mm + Sm sclerotized, Sm rectangular (Fig. 1E). Wing length: fore, 5.90; hind, 5.10. Wing venation (both wings): type $\mathrm{c}, \mathrm{Rs}+\mathrm{Ma}$ and $\mathrm{Rs}$ well developed, first 1/5 of Ma conspicuous, the rest inconspicuous, $\mathrm{Mp}$ and $\mathrm{Cua}$ inconspicuous. Anal diffuse. Cross veins (both wings): 1 R1-Rs. Hind basitarsus (Fig. 1D): length 0.53 , ratio 0.24 , chaetotaxy: $5-6 \mathrm{P}(1: 3 \mathrm{~A}) ; 7-8 \mathrm{P}(2: 3 \mathrm{~B})$; 7R(1:5B); 1R(1:4A); 20V(1:2B). Terminalia (Fig. 1B, C): 10Rp1 with the apex lightly expanded and truncate, with many longitudinal carinae. 10Lp1 basally broader with acute apex. Hp longer than $\mathrm{H}, \mathrm{Hp}$ with apex acute and directed to the left side. $\mathrm{H}$ with a depressed area next to the anterior margin of Rpp. Lpp and Rpp well developed, sclerotized and globose; both are prolonged to the dorsal region, hence, conspicuous in the dorsal view. Longitudinal ratio of LC2/LC1: 0.91 .

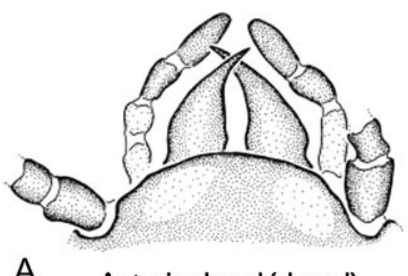

A

Anterior head (dorsal)

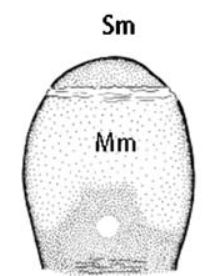

E
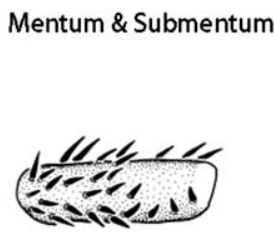

D Third basitarsus

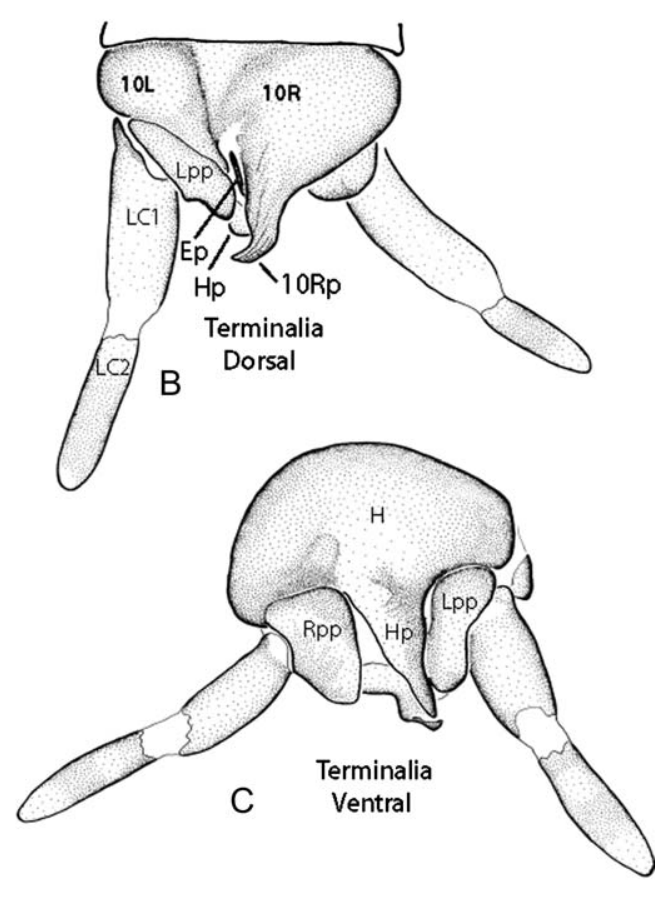

Fig. 1. Traits of male Saussurembia calypso sp.n., holotype, type locality: Monas Trinidad. A, Mouthparts; B, C, terminalia; D, hind basitarsus; E, submentum and mentum of labium (see text for abbreviations). 
Female (paratype). Colour (Fig. 2): head yellowishorange. Antenna: antennites 1 and 2 yellowish-white, the rest lighter brown. Prothorax yellowish-white. Mesothorax dark brown. Metathorax and first four tergites light yellowishbrown with two lateral small circular areas (per tergite) whitish and with whitish bands between the sternites; the other abdominal tergites and sternites dark brown with some reddish pigmentation. Basal cerci brownish white (as in males) and apical cerci light brown. Forelegs yellowishorange; middle and hindlegs light yellowish-brown with the exception of dark brown tibia.

Total length: 9.80. Head: width/length: 0.82; OR: 0.83 . Hind basitarsus (Fig. 3C): length: 0.38; width/length: 0.33;

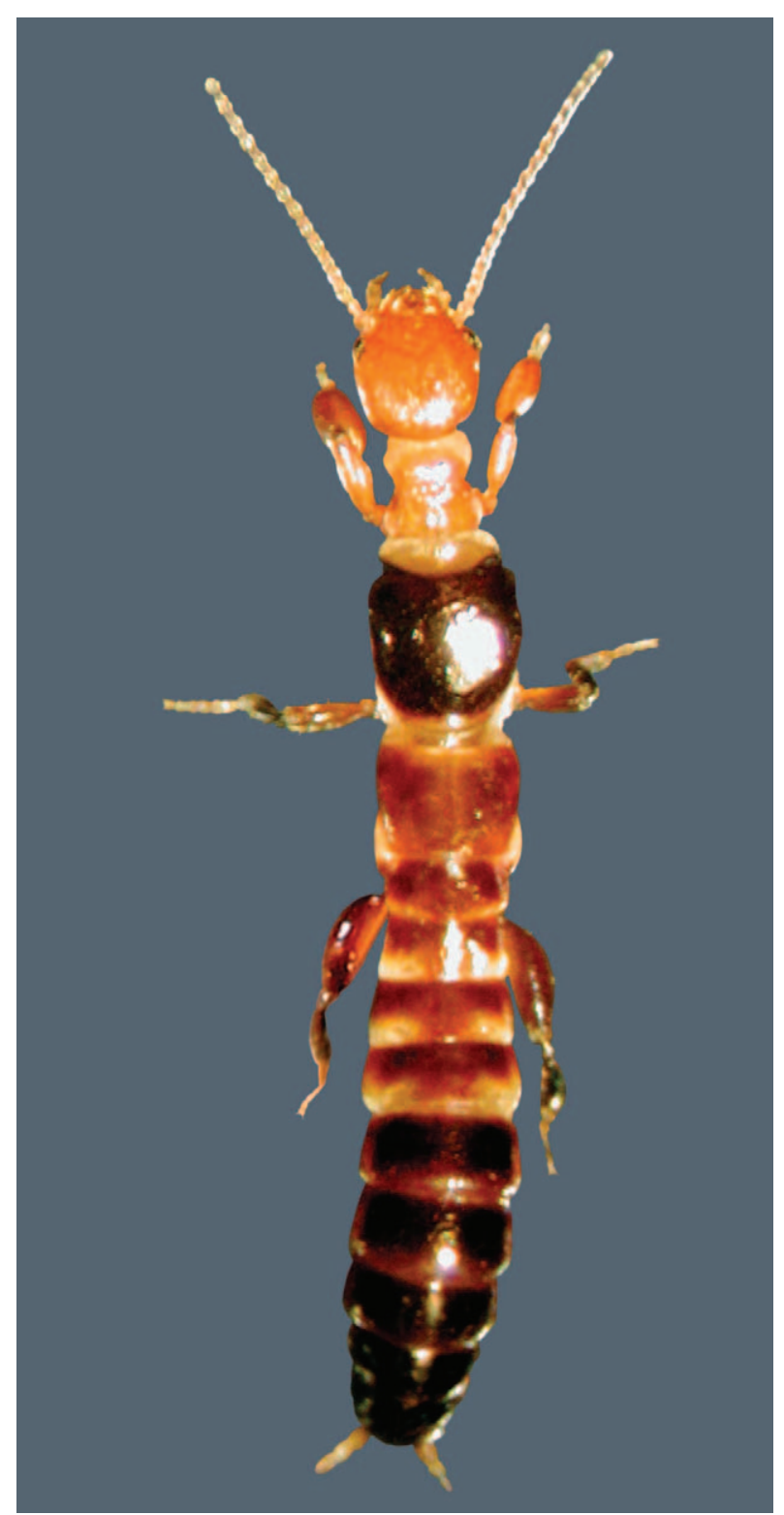

Fig. 2. Female Saussurembia calypso sp.n. photographed live at Santa Clara University. She is approximately $9.8 \mathrm{~mm}$. chaetotaxy: $2 \mathrm{R}(1: 4 \mathrm{~A}) ; 7 \mathrm{~A}(1: 2 \mathrm{~B}) ; 5 \mathrm{P}(1: 3 \mathrm{~A}) ; 8 \mathrm{P}(2: 3 \mathrm{~B})$ in two rows; $18-22 \mathrm{~V}(2: 3 \mathrm{~B})$. Terminalia as in Fig. $3 \mathrm{~B}$.

Type material. Holotype ô Saussurembia calypso sp.n. Edgerly 2003, TRINIDAD: Monas Island $\left(61^{\circ} 40^{\prime} \mathrm{W}\right.$; $\left.10^{\circ} 42^{\prime} \mathrm{N}\right), 10$. vii. 2003 (Edgerly); Deposited in the collection of the Sección de Entomología, Instituto y Fundación Miguel Lillo, Tucumán, Argentina (IFML). Paratypes, TRINIDAD, three males and four females same data as holotype. Matured in culture (iv.2004). All the specimens are in alcohol.

Biology. Specimens were collected from a colony on the bark of an ornamental tree in a manicured lawn area around coastal homes on Monas Island between Trinidad and mainland Venezuela. Nearby palms and fruit trees harboured colonies of the exotic Asian species Oligotoma saundersii (Oligotomidae), suggesting that embiids have hitched rides with people to the small island. Therefore, the population of $S$. calypso may also have been introduced there, although natural affiliation with Venezuela is also likely as the genus is South American (Ross, 1972). Their silk is fashioned into tubes that follow the fissures of rough bark. In culture jars at Santa Clara University, they produced layers of tubes affixed to the smooth surface of the jar. Individuals run back and forth through these tubes, and numerous individuals of all ages crowd together in the jumble of tubes. Nymphs and adult females are very active, often wandering outside of their silk. This behaviour is considered to be unusual because embiids generally hide within silk domiciles. Their conspicuous behaviour is intriguing given their banded orange and brown coloration pattern (Fig. 2), suggestive of warning coloration or mimicry known in other insects. Experimentation is needed to confirm the function of their colour pattern.

Eggs and oviposition. Each creamy-white egg (Fig. 3A), approximately $1.05 \mathrm{~mm}$ in length, is roughly cylindrical, rounded on one end, and curved slightly along one side (see Fig. 4 for side view of egg). The operculum extends about one-third the length of the egg. The cuticular ridge of the operculum is a distinct white line, smooth with the surface of the egg (unlike the more conspicuously raised ridge seen in Antipaluria urichi and others) (see Part 2). The egg of $S$. calypso closely resembles that of congener $S$. davisi (see below) and another anisembiid, Anisembia texana (drawings in Melander, 1903).

A 1-day census of laboratory cultures at Santa Clara University revealed that eggs were widely scattered on twenty-three of the fifty pieces of bark used as habitat [bark averaged $53 \pm 6.1 \mathrm{~cm}^{2}$ (SE) in size]. Of the eighty-eight eggs found, sixty-one $(69 \%)$ were solitary and fourteen $(16 \%)$ were in pairs $(10 \%$ were in groups of three and $4.5 \%$ in groups of four). In addition, females $(n=17)$ separated into a group of five and two groups of six, and closely observed for 1 month, showed the same pattern as detected in the cultures. These females laid twenty-two eggs: thirteen eggs were alone, and the others were found in separate groups of 


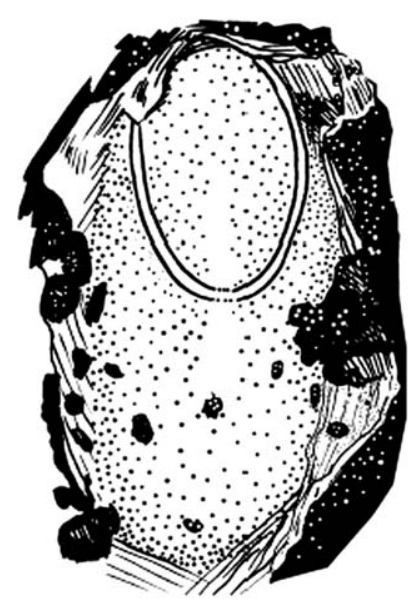

A Egg in situ

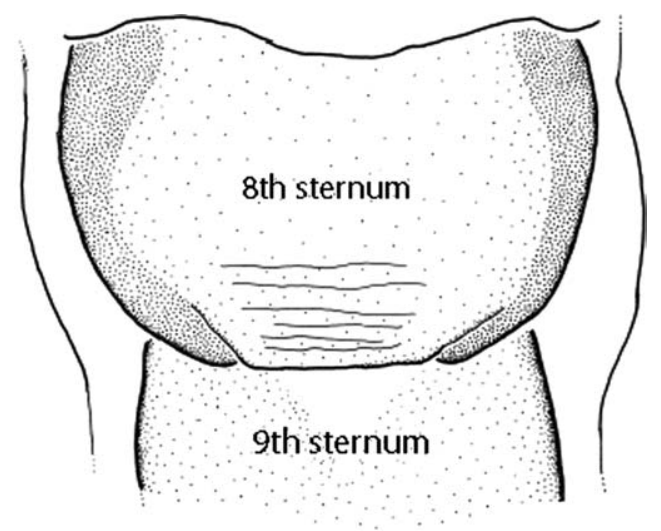

B Terminalia Ventral

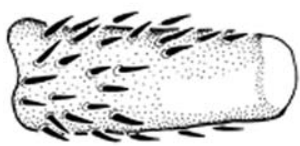

C Third basitarsus
Fig. 3. Traits of female Saussurembia calypso sp.n., paratype and an egg. A, Egg (length, $1.05 \mathrm{~mm}$ ); the egg is shown with a thin silk coating here and there and with patches of macerated material, which are collected by the mother; the egg was traced from a photograph taken through a dissecting microscope with a SonyDFWilink digital camera; B, terminalia (ventral); $\mathrm{C}$, hind basitarsus. two, three and four. Females were not seen attending the eggs, and when their silk was breached with forceps, none of them returned to repair the silk as other embiids are known to do. These females loosely attached the eggs to bark, stitched them into silk tunnels or buried them within crevices of the bark. Macerated material was sometimes stitched around the egg in the silk (as in Fig. 3A), but when the silk was moved, the macerated material was also easily dislodged. In other species, the treatment of eggs by females is different. For example, females often adhere gathered materials or thick silk to the egg's surface, and assemble them into organized batches, sometimes affixed tightly to the substrate (see references in Edgerly, 1997; Ross, 2000). Interestingly, the treatment of eggs by another anisembiid, A. texana, is similar to that of S. calypso; Melander (1903) observed captive females fastening single eggs loosely into their silk. Choe (1994) noted that $A$. texana females $(n=3)$ coated their eggs with scraped debris. Each egg was suspended in a silk sheath that separated the egg from the interior of the silk tunnel.

Our observations suggest that $S$. calypso oviposit, nestle the egg into silk or a crevice, but then leave it. Because eggs are scattered, they may not provide an opportunity for eggguarding by the mother. Embiids are termed subsocial (see references in Edgerly, 1987) because they exhibit maternal care. Indeed, the plesiomorphic Antipaluria urichi, the subject of in-depth investigations (for example, Edgerly, 1987, 1988), produces complex egg masses with layers of protective coatings that thwart parasitic wasps. Antipaluria urichi lays numerous eggs per batch (mean of fifty-three in the field, up to 117 seen in the laboratory at Santa Clara University), and exhibits a suite of behaviours associated with egg-guarding.
The significance of the maternal trait for the order and the lack of obvious egg-guarding in S. calypso warrant further investigation. Choe (1994) investigated this behaviour in A. texana and discovered that solitary females with eggs became highly aggressive towards other embiids, suggesting that maternal care exists in this closely related species despite the similar unorganized placement of their eggs.

\section{Part 2. Embiid eggs}

Despite Ross's (2000: 13) claim for embiids that 'eggs of all species are remarkably similar', the striking differences between S. calypso eggs and those of Antipaluria urichi, and the striking similarities to another anisembiid egg (A. texana), suggested to us that egg shapes may reflect phylogeny. Ovaries (where the eggs are formed) are adult structures that may express more about phylogeny than is apparent in a female's nymphoid body shape. Therefore, egg morphology may provide clues about a maternal embiid's taxon, which was heretofore almost impossible to determine without access to associated males (often rare or even missing completely from colonies in the field).

\section{Taxon sampling}

To conduct our analysis, we collected eggs and adult females from cultures at Santa Clara University and photographed them with a Sony DFWilink digital camera connected to an Olympus dissecting microscope. Measurements were made using ImageJ 1.3 software; the scale was 
Fig. 4. Eggs (from top to bottom) of Antipaluria urichi (Clothodidae), Saussurembia calypso sp.n. (Anisembiidae), Notoligotoma hardyi (Notoligotomidae) and Australembia incompta (Australembiidae). Black lines are guidelines added to highlight the curvature of the upper and lower edges of the egg (characters 1 and 2). The broken line shows the operculum angle (character 8 ), measured digitally (see text).

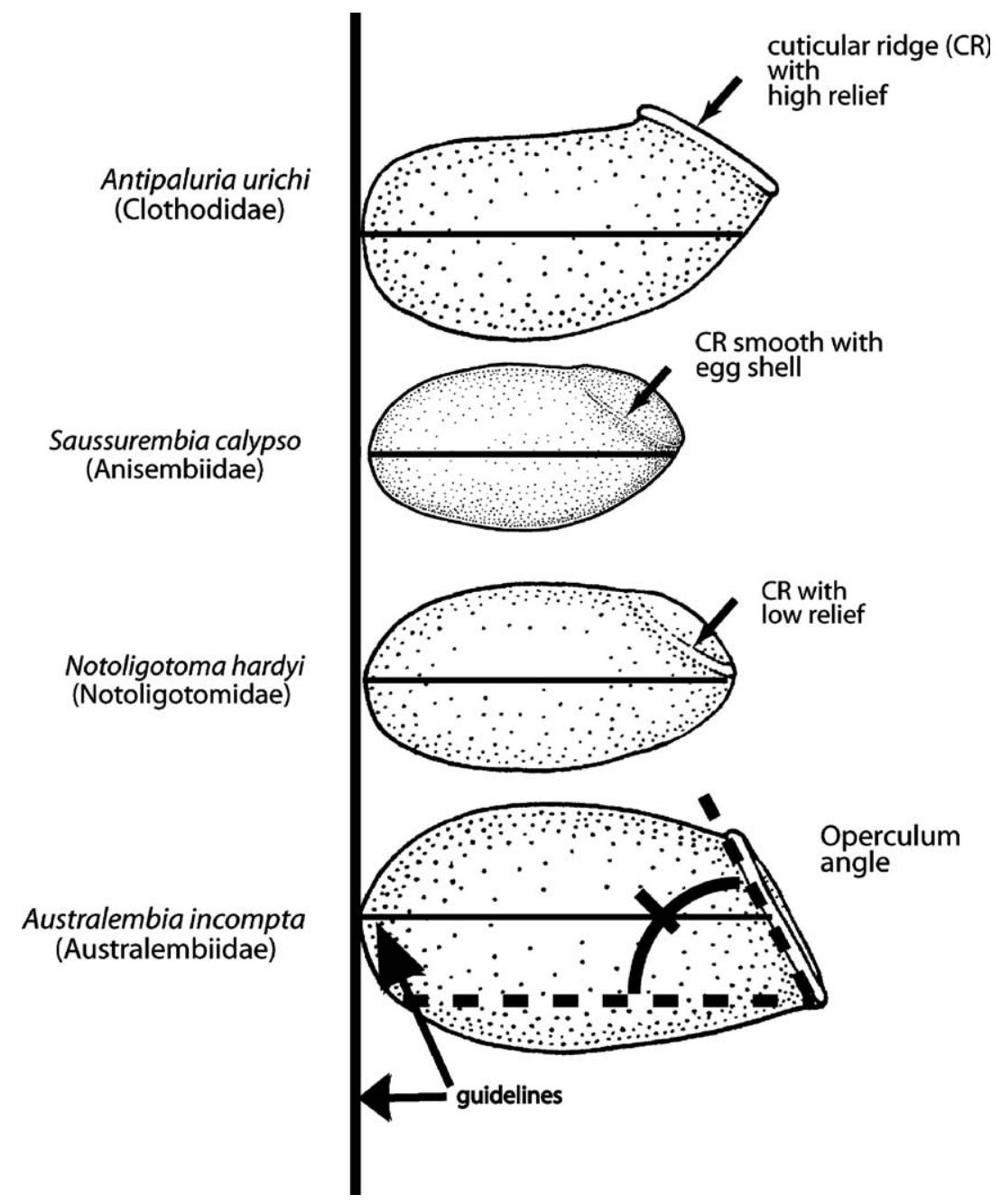

upper curve is: 0 , fairly straight line; 1, angled upward gradually then meets operculum with short neck; 2 , bowed, curves up and then down again.

Character 2. Curvature of edge viewed from the side (side opposite to operculum) (Fig. 4): 0, fairly straight; 1, slightly bowed; 2, strongly bowed.

Character 3. Height of the cuticular ridge of the operculum (Fig. 4): 0, high relief (the ridge is distinct and raised up); 1, low relief; 2, almost smooth with egg shell.

Character 4. Size of egg relative to female head width; the ratio between the area of the side of the egg and the head width of the female, measured along a line through the widest point of, and including, the eyes.

Character 5. Egg shape: described as the ratio between the width one-quarter of the way down the egg and the width onequarter of the way up from the bottom (Fig. 5); this measurement depicts a range of forms from oval to cup shaped.

Character 6. Operculum shape (Fig. 5): the ratio between the width at the halfway point and the length. With this measurement, shapes ranging from oval to circular are described. Character 7. Relative size of the operculum: the ratio between the length of the operculum and the long axis of the egg. 


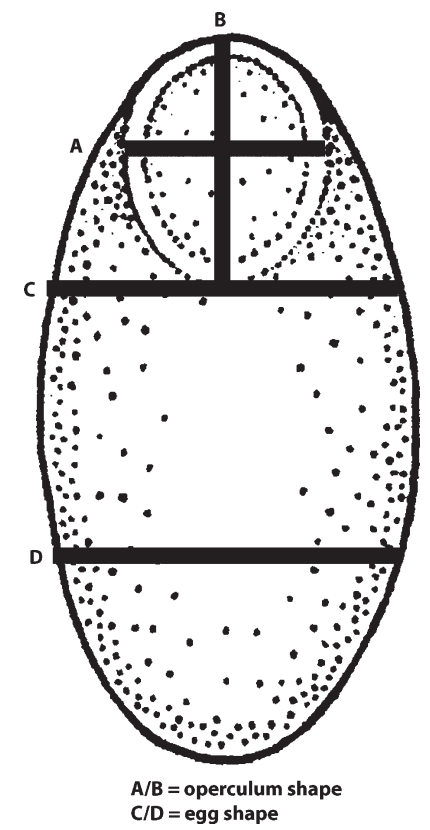

Fig. 5. The egg shape (character 4) was determined by the ratio of lines $\mathrm{C}$ to $\mathrm{D}$, and the operculum shape (character 5) was determined by the ratio of lines $\mathrm{A}$ to $\mathrm{B}$. Traits were optimized onto the cladogram of adult morphology.

Character 8. Operculum angle (Fig. 4): measured as the angle between a line perpendicular to the base of the egg and the operculum ridge. The opening position of the operculum could be from across to along the long axis of the egg.

Character 9. Surface of the operculum viewed from the side (Fig. 4): 0, convex, it is visible; 1, concave, inconspicuous. Characters 1, 2, 3 and 9 are discrete and codified as additive; the others are continuous characters (see data matrix in Table 1). Measurements include the mean and its standard deviation, except for character 4.

\section{Cladistic analysis}

Two very simple means exist to test the extent to which egg features are explained by common ancestry. Most logically, egg characters are optimized onto a phylogeny obtained from another source (i.e. reduced consensus tree of adult morphological data; Szumik et al., 2003). New characters that map well onto the cladogram would be significant. Alternatively, the cladogram obtained from the egg data matrix can be compared with that obtained from adult morphological data. Again, the similarity between the two hypotheses, with evidence analysed separately, indicates concordance. Optimization of the character traits onto the consensus tree of Szumik et al. (2003), as well as tree searches for the egg data matrix, were carried out with the program TNT (Goloboff et al., 2004; see reviews of the program in Hovenkamp, 2004 and Meier \& Ali, 2005). To check the

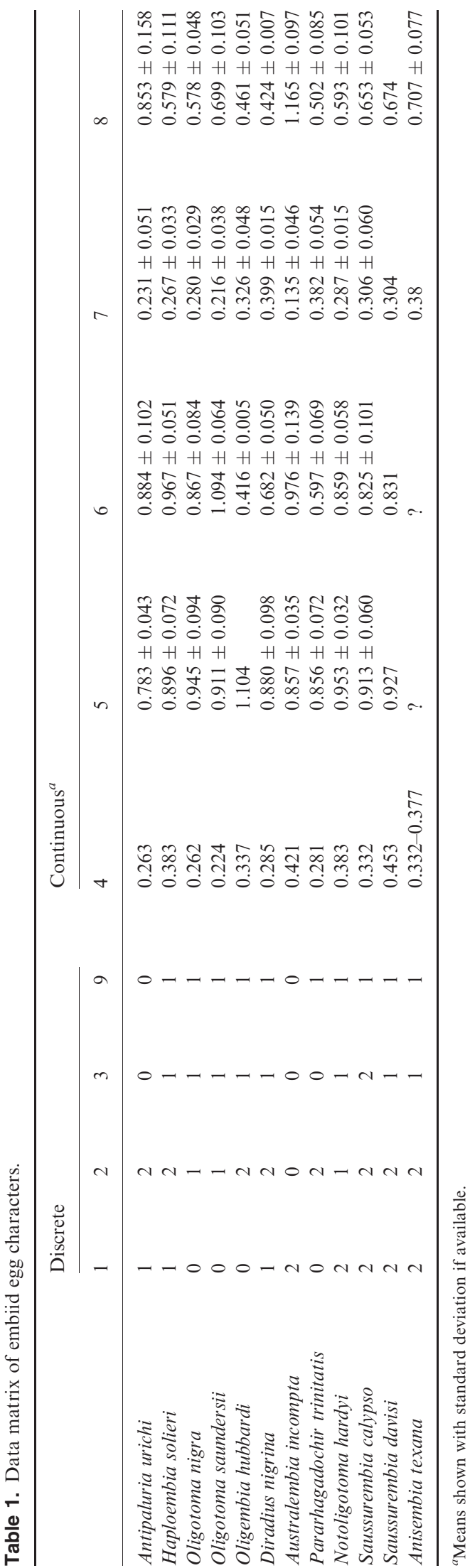

(C) 2007 The Authors Journal compilation (C) 2007 The Royal Entomological Society, Systematic Entomology, 32, 387-395 
concordance between the two hypotheses, the agreement subtree was calculated for all trees (Goloboff et al., 2004).

\section{Results and discussion}

Although the nine characters map imperfectly on the cladogram of adult morphology, there is some congruence (see Figs 6 and 7). States 1 and 2 of the egg curvature (character 1) map perfectly, whereas state 0 appears three times (Fig. 6A). The oval operculum (character 6) appears twice, once for the family Teratembiidae and another occurrence for Pararhagadochir, whereas the other species have a somewhat circular operculum (Fig. 6B). The opercular angle (character 8) decreases through the cladogram with only a great increment in Australembidae (which is known as a neotenic family; Fig. 6C). The convexity of the operculum (character 9) surprisingly appears twice (Fig. 7A), once in Clothodidae (considered as the 'most plesiomorphic' Embioptera) and in the neotenic Australembiidae.

The cladogram obtained from the egg characters (Fig. 7B) fails to recover some groups, such as Oligotomidae, Teratembiidae, Saussurembia, etc.; however, if this cladogram is compared with the cladogram based on adult morphology (Szumik et al., 2003) using agreement subtrees
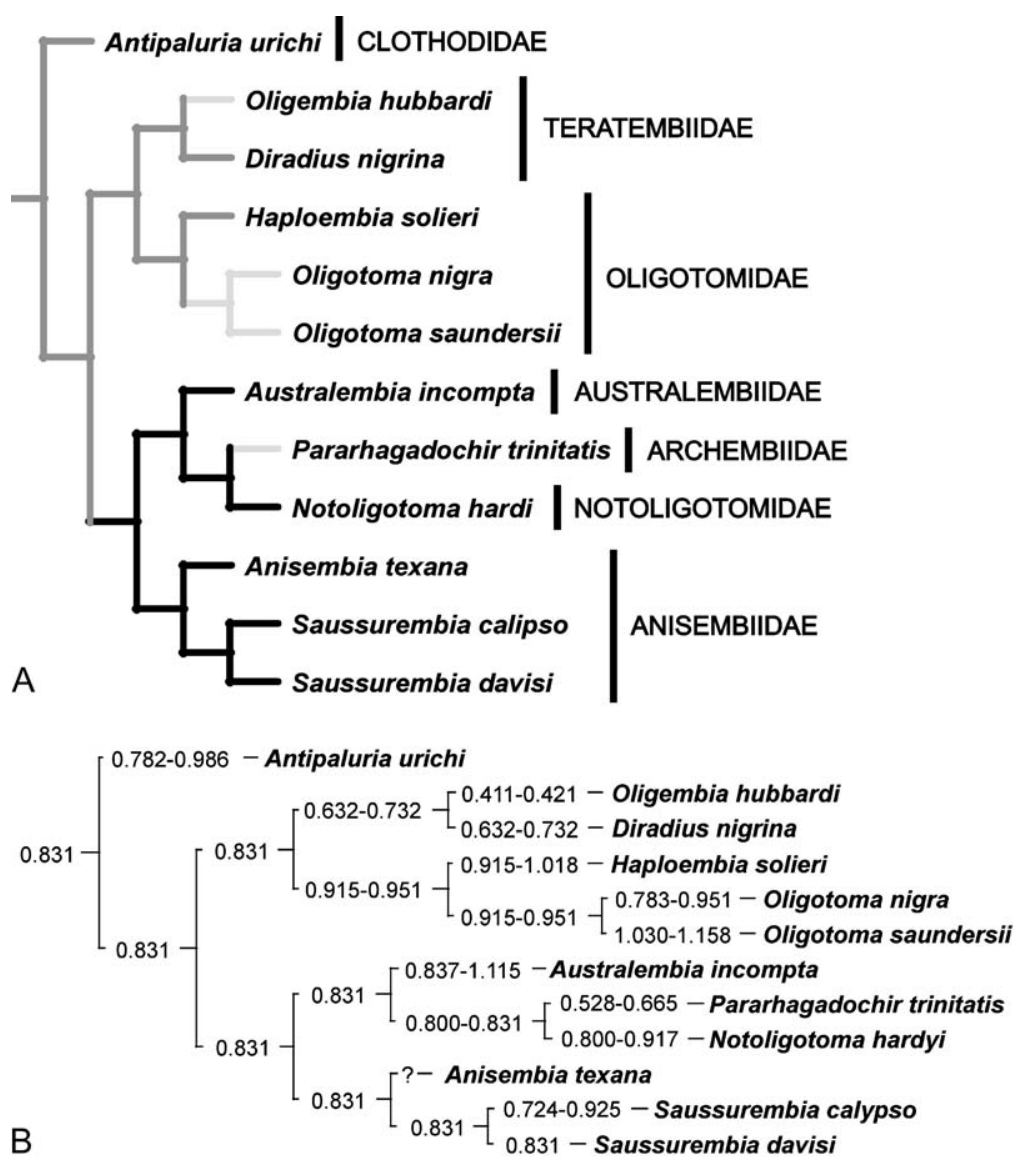

Fig. 6. A, Curvature of upper edge of egg (character 1) optimized onto the cladogram of adult morphology. Light grey, fairly straight line; dark grey, angled upward gradually then meets operculum with short neck; black, bowed, curves up and then down again. B, Operculum shape (character 6) optimized onto the cladogram of adult morphology. C, Operculum angle (character 8) optimized onto the cladogram of adult morphology.

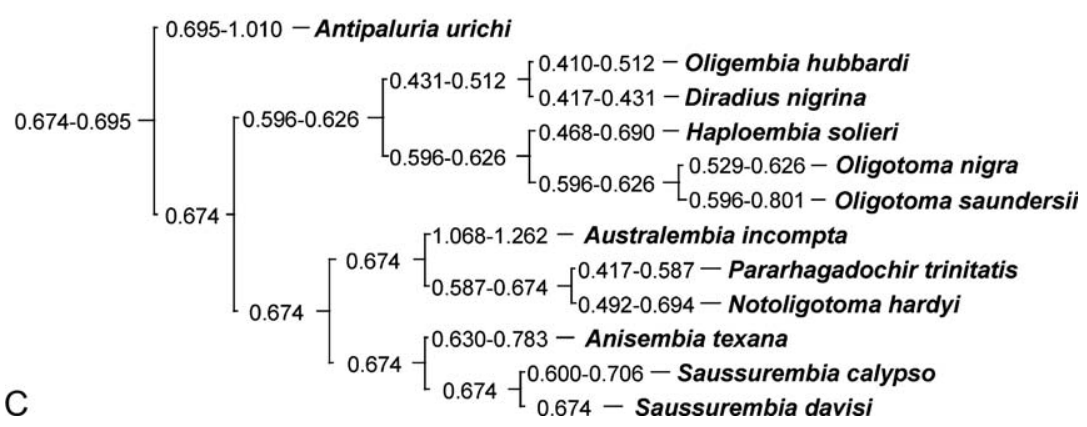


(Fig. 7C), eight of the twelve taxa appear, which shows a fairly good concordance.

The traditional classification of the order and the alpha taxonomy, according to Ross (1972), were constructed solely for the male terminalia. Although there have been serious attempts to include other traits of the female terminalia, male and female chaetotaxy, wing traits, and male and female cephalic structures in the higher classi- fication and alpha taxonomy, because they are potentially useful (Szumik, 1996; Szumik et al., 2003), more comparative studies on new characters of behaviour or nymph instars would be desirable. The promise of this first attempt to analyse the systematic utility of the egg within a phylogenetic framework suggests that a concerted effort to collect and describe embiid eggs would be valuable.
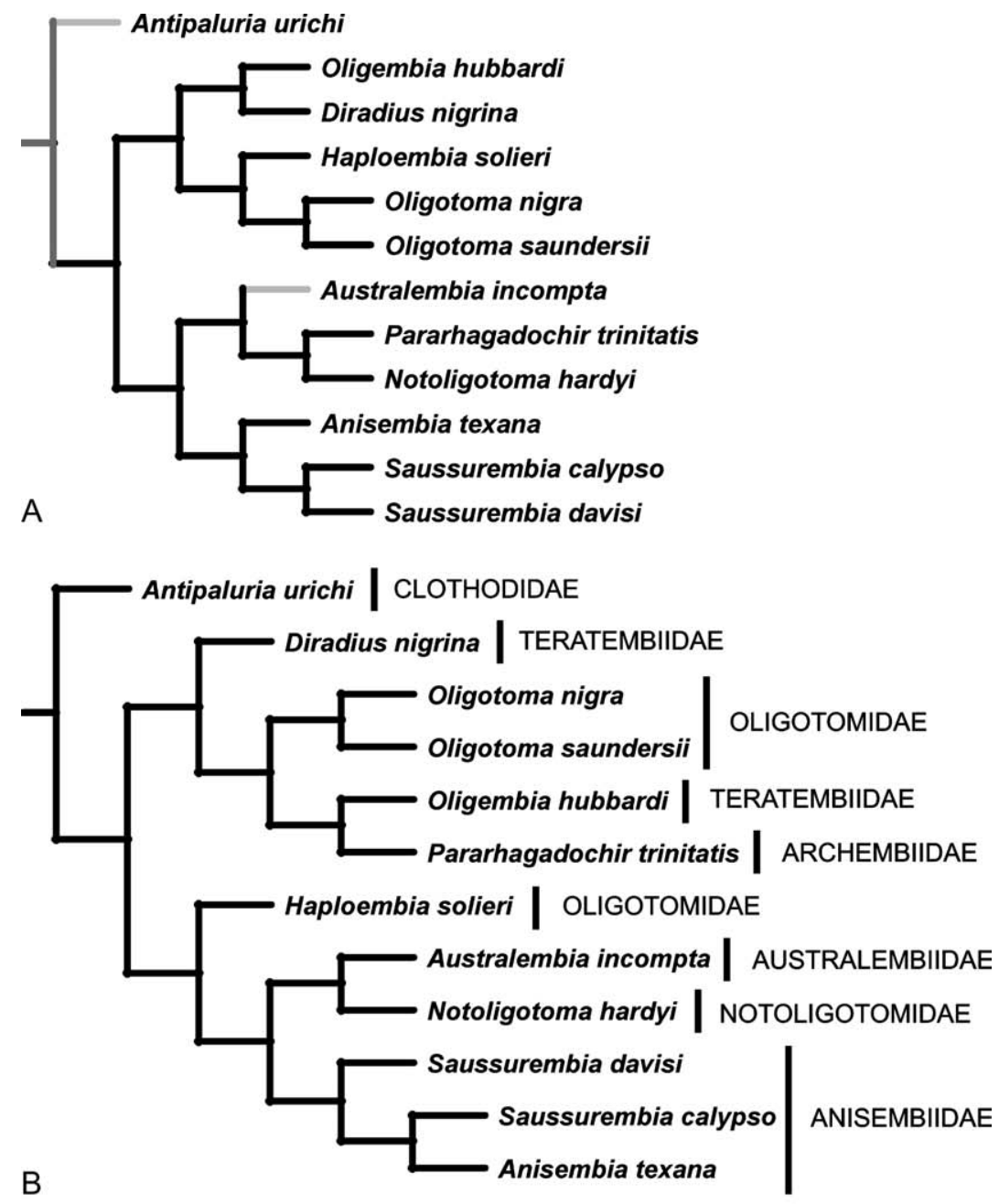

B Antipaluria urichi

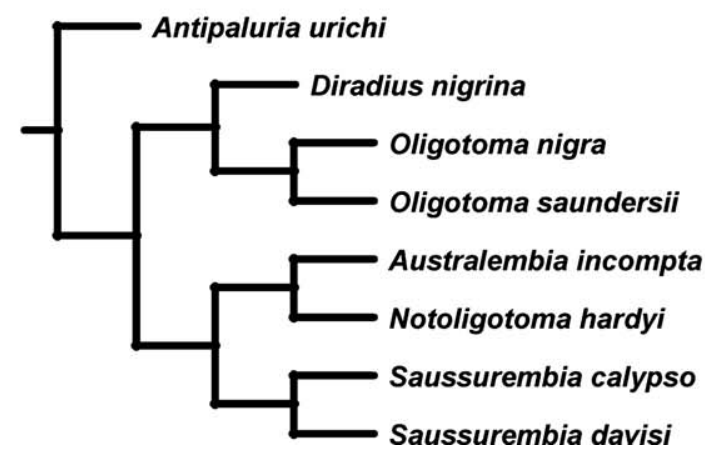

Fig. 7. A, Surface of the operculum viewed from the side (character 9) optimized onto the cladogram of adult morphology. Light grey, convex; black, concave; darker grey, ambiguous. B, Cladogram obtained from egg data matrix. C, Agreement subtree of egg traits and adult morphology. 


\section{Acknowledgements}

This study was supported by the Agencia de Promoción Científica y Tecnológica PICT2002-01-12605 (CAS) and by a grant from the Howard Hughes Medical Institute to Santa Clara University (Community of Science Scholars Initiative). The authors thank James M. Carpenter, Luisa Montivero and Pablo Goloboff who provided useful comments and criticisms.

\section{References}

Borror, D.J. (1960) Dictionary of Word Roots and Combining Forms. Mayfield Publishing Co., Mountain View, California.

Choe, J. (1994) Communal nesting and subsociality in a webspinner, Anisembia texana (Insecta: Embiidina: Anisembiidae). Animal Behaviour, 47, 971-973.

Davis, C. (1939) Taxonomic notes on the Order Embioptera. Part XIV. The identity of Embia ruficollis De Saussure and of Oligotoma venosa Banks. Proceedings of the Linnean Society of New South Wales, 64, 572-575.

Edgerly, J.S. (1987) Maternal behaviour of a webspinner (Order Embiidina). Ecological Entomology, 12, 1-11.

Edgerly, J.S. (1988) Maternal behaviour of a webspinner (Order Embiidina): mother-nymph associations. Ecological Entomology, 13, 263-272.

Edgerly, J.S. (1997) Life beneath silken walls: a review of the primitively social Embiidina. Social Behavior in Insects and Arachnids (ed. by J.C. Choe and B.J. Crespi), pp. 14-25. Cambridge University Press, Cambridge.
Goloboff, P., Farris J. \& Nixon K. (2004) TNT: Tree Analysis Using New Technology, Version 1.0. Program and documentation available from the authors and at: URL www.zmuc.dk/public/ phylogeny [accessed on 15 March 2006].

Grimaldi, D. \& Engel M.S. (2005) Evolution of Insects. Cambridge University Press, New York.

Hovenkamp, P. (2004) Review of TNT - Tree Analysis Using New Technology, Version 1.0. Cladistics, 20, 378-383.

Meier, R. \& Ali, F.B. (2005) The newest kid on the parsimony block: TNT (Tree analysis using new technology). Systematic Entomology, 30, 179-182.

Melander, A.L. (1903) Notes on the structure and development of Embia texana. Biology Bulletin, 4, 99-118.

Ross, E.S. (1972) New South American Embioptera. Studies in Neotropical Fauna, 7, 133-146.

Ross, E.S. (2000) EMBIA, Contributions to the biosystematics of the Insect Order Embiidina. Part 2. A review of the biology of Embiidina. Occasional Papers of the California Academy of Sciences, 149.

Szumik, C.A. (1991) Two new species of Teratembiidae (Embiidina) from Argentina. Journal of the New York Entomological Society, 99, 611-621.

Szumik, C.A. (1996) The higher classification of the order Embioptera: a cladistic analysis. Cladistics, 12, 41-64.

Szumik, C.A. (1998) Two new neotropical genera of Embiidae (Embioptera, Insecta). Journal of the New York Entomological Society, 105, 140-153.

Szumik, C.A., Edgerly J. \& Hayashi C. (2003) Phylogenetics of Embioptera (= Embiidina). Entomologische Abhandlungen, 61, 131.

Accepted 22 December 2006 
Copyright of Systematic Entomology is the property of Blackwell Publishing Limited and its content may not be copied or emailed to multiple sites or posted to a listserv without the copyright holder's express written permission. However, users may print, download, or email articles for individual use. 